\title{
Breakdown of Stokes-Einstein relation in the supercooled liquid state of phase change materials
}

\author{
Gabriele C. Sosso ${ }^{1}$, Jörg Behler², and Marco Bernasconi讯 \\ 1 Dipartimento di Scienza dei Materiali, Università di Milano-Bicocca, Via R. Cozzi 53, I-20125 Milano, Italy and \\ 2 Lehrstuhl für Theoretische Chemie, Ruhr-Universität Bochum, \\ Universitätsstrasse 150, D-44780 Bochum, Germany
}

\begin{abstract}
The application of amorphous chalcogenide alloys as data-storage media relies on their ability to undergo an extremely fast (10-100 ns) crystallization once heated at sufficiently high temperature. However, the peculiar features that make these materials so attractive for memory devices still lack a comprehensive microscopic understanding. By means of large scale molecular dynamics simulations, we demonstrate that the supercooled liquid of the prototypical compound GeTe shows a very high atomic mobility $\left(D \sim 10^{-6} \mathrm{~cm}^{2} / \mathrm{s}\right.$ ) down to temperatures close to the glass transition temperatures. This behavior leads to a breakdown of the Stokes-Einstein relation between the selfdiffusion coefficient and the viscosity in the supercooled liquid. The results suggest that the fragility of the supercooled liquid is the key to understand the fast crystallization process in this class of materials.
\end{abstract}

\section{INTRODUCTION}

Phase-change materials based on chalcogenide alloys are attracting a lot of interest due to their ability to undergo reversible and fast transitions between the amor-

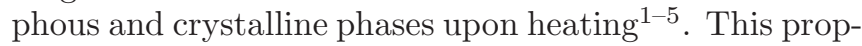
erty is exploited in rewriteable optical media (DVD, Blu-Ray Discs) and phase change non volatile memories $(\mathrm{PCM})$. The strong optical and electronic contrast between the crystal and the amorphous allows discriminating between the two phases that correspond to the two states of the memory. The PCM devices, first proposed by Ovshinsky in the late 1960's ${ }^{6}$, offer extremely fast programming, extended cycling endurance, good reliability and inexpensive, easy integration. A PCM is essentially a resistor of a thin film of a chalcogenide alloy (typically $\mathrm{Ge}_{2} \mathrm{Sb}_{2} \mathrm{Te}_{5}, \mathrm{GST}$ ) with a low field resistance that changes by several orders of magnitude across the phase change. In memory operations, cell read out is performed at low bias. Programming the memory requires instead a relatively large current to heat up the chalcogenide and induce the phase change, either the melting of the crystal and subsequent amorphization (reset) or the recrystallization of the amorphous (set).

The key property that makes these materials suitable for applications in PCM is the high speed of the transformation which leads to full crystallization on the time scale of 10-100 ns upon Joule heating. What makes some chalcogenide alloys so special in this respect and so different from most amorphous semiconductors is, however, still a matter of debate.

In this paper, we demonstrate by means of atomistic molecular dynamics (MD) simulations that the phase change compound GeTe displays a very high mobility in the supercooled liquid phase down to very low temperatures $T$ leading to a breakdown of the Stokes-Einstein relation $D \propto \frac{T}{\eta}$ (SER) between diffusivity $D$ and viscosity $\eta$. The breakdown of SER in the supercooled liquid phase, which is typical of a fragile liquid $\underline{\underline{Z}}$, is actually the key to understand the fast crystallization behavior of these materials as discussed below.

Supercooled liquids are classified as fragile or strong on the basis of the temperature dependence of their viscosity $\underline{\underline{7}}$. An ideal strong liquid shows an Arrhenius behavior of the viscosity $\eta$ from the melting temperature $\mathrm{T}_{m}$ down to the glass transition temperature $\mathrm{T}_{g}$. On the contrary, in a fragile liquid $\eta$ follows an Arrhenius behavior only above a cross-over temperature $\mathrm{T}^{*}$ below which a Vogel-Tammann-Fulcher (VTF) function $\eta=\eta_{o} \exp \left(\frac{E}{k_{B}\left(T-T_{o}\right)}\right)$ is customarily used to reproduce the data with $\eta_{o}, E$ and $\mathrm{T}_{o}$ as fitting parameters ${ }^{7}$. The viscosity in a fragile liquid shows a steep rise by approaching $\mathrm{T}_{g}$ which according to the SER would lead to a strong decrease in the atomic mobility. On the other hand, the self diffusion coefficient $D$ controls both the speed of crystal growth $u$ and the steady state nucleation rate $I_{s s}$. In fact, classical nucleation theory $\underline{5.8}$ predicts that $u \propto$ $D\left(1-\exp \left(-\Delta G /\left(k_{B} T\right)\right)\right.$ and $I_{s s} \propto D \exp \left(-G_{c} /\left(k_{B} T\right)\right)$ where $\Delta G$ is the free energy difference between the liquid (or amorphous) and the crystalline phases and $G_{c}$ is the formation free energy of the critical nucleus given in turn by $G_{c}=16 \pi \sigma^{3} /\left(3 \Delta G^{2}\right)$ with $\sigma$ interface energy. $\Delta G$ is the driving force for crystallization that decreases by increasing temperature and finally vanishes at $\mathrm{T}_{m}$. In phase change materials, due to the breakdown of SER, the diffusivity can be very high just above $\mathrm{T}_{g}$ in spite of a large viscosity. Consequently $D$ can reach high values at temperatures much lower than $\mathrm{T}_{m}$ where a large driving force for crystallization is present.

A breakdown of SER in GST has actually been suggested by recent measurements of the crystallization rate

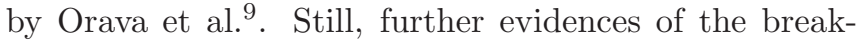
down of SER are needed to support the crucial assumptions made by Orava et al. to infer the viscosity from their only data of differential scanning calorimetry.

To this aim we performed MD simulations by using a classical interatomic potential ${ }^{10}$ we generated by fitting a large database of density functional energies by means of 
the Neural Network (NN) method introduced in Ref 11 . So far we have restricted ourselves to the binary compound GeTe which is also under scrutiny for applications in PCM and which shares most of the properties with the more commonly used GST ${ }^{4,12,13}$. The NN potential displays an accuracy close to that of the underlying density functional theory (DFT) framework at a much reduced computational load that scales linearly with the size of the system. It allows us to simulate several thousands of atoms for tens of ns, which is well beyond present-day capabilities of DFT molecular dynamics.

\section{COMPUTATIONAL DETAILS}

The NN interatomic potential of GeTe was obtained in Ref $\stackrel{10}{0}$ by fitting a huge database of DFT energies by means of the method introduced by Behler and Parrinello $\underline{\underline{11}}$. The database consists of the total energies of about 30000 configurations of 64-, 96-, and 216-atom supercells computed by employing the the Perdew-BurkeErnzerhof (PBE) exchange and correlation functional ${ }^{14}$ and norm conserving pseudopotentials. The NN potential displays an accuracy close to that of the underlying DFT-PBE framework whose reliability in describing structural and dynamical properties of GeTe and other phase change materials has been validated in several previous works by our $\stackrel{10,13,15}{1}$ and other groups $\frac{12}{12}$.

The simulations were performed with the NN code RuNNer ${ }^{16}$ and a 4096 -atom cubic supercell by using the DL_POLY 17 code as MD driver. The time step was set to $2 \mathrm{fs}$, and constant temperature was enforced by a stochastic thermostat 18 .

It turned out that in order to reproduce the equilibrium density of the liquid at $\mathrm{T}_{m}$, an empirical van der Waals (vdW) correction had to be added to the NN potential. This was done by using the scheme proposed by Grimme $\frac{19}{2}$ with the $s_{6}$ parameter tuned to 0.55 to reproduce the experimental equilibrium volume of the liquid at $\mathrm{T}_{m} \underline{20}$. The experimental equilibrium volume of the amorphous and crystalline phases are instead well reproduced by the NN potential without the need of the $\mathrm{vdW}$ interaction $\underline{10}$. The inability of the NN potential in reproducing the equilibrium volume of the liquid can be traced back to the presence of nanovoids in the melt $\frac{12}{2}$. In the liquid the nanovoids can coalesce and increase in size by decreasing the density which results into a reduced tensile stress upon expansion. This effect is hindered by vdW interactions. Nanovoids are also present in the amorphous phase $\frac{12}{2}$, but their distribution can not change with temperature because of the low atomic mobility in the amorphous phase. The calculated linear thermal expansion coefficient $\left(\alpha=\frac{1}{3 V} \frac{\partial V}{\partial T}\right)$ of the liquid at $\mathrm{T}_{m}$ with the $\mathrm{NN}+\mathrm{vdW}$ potential turned out to be $4.73 \cdot 10^{-5} \mathrm{~K}^{-1}$ to be compared with the experimental value of $3.73 \cdot 10^{-5}$ $\mathrm{K}^{-120}$.

The added vdW interaction acts just as a volume dependent term in the equation of state of the liquid but it is not included in the MD simulations discussed below. DFT calculations of the self-diffusion coefficient at two selected temperatures were performed by molecular dynamics simulations within the Born-Oppenheimer approach by using the code $\mathrm{CP}_{2} \mathrm{~K}^{21}$; Kohn-Sham orbitals were expanded in a Triple-Zeta-Valence plus Polarization Gaussian-type basis set and the charge density was expanded in a planewave basis set with a cut-off of $100 \mathrm{Ry}$ to efficiently solve the Poisson equation within the Quickstep scheme 21 . Goedecker-type pseudopotentials 22 with four and six valence electrons were used for $\mathrm{Ge}$ and $\mathrm{Te}$, respectively. Brillouin Zone integration was restricted to the $\Gamma$ point of the 216 -atom supercell. The same framework was used in our previous DFT molecular dynamics simulations of $\mathrm{GeTe} \underline{\underline{10}}, \underline{13}$.

\section{RESULTS}

To study the properties of the supercooled liquid, we first assessed the ability of the NN potential, and thus of the underlying DFT-PBE framework, to reproduce $\mathrm{T}_{m}$. The melting temperature was computed by means of thermodynamic integration ${ }^{23}$ that yielded $\mathrm{T}_{m}=1001$ $\mathrm{K}$ very close to the experimental value at normal pressure of $998 \mathrm{~K}^{24}$. To obtain $\mathrm{T}_{m}$, we first computed the difference in the Helmholtz free energy $F$ between the NN system and a reference system for which an analytic expression for $F$ is known, at a given temperature $T^{\prime}$ and density $\rho^{\prime}$. Namely

$$
F_{N N}\left(T^{\prime}, \rho^{\prime}\right)-F_{r e f}\left(T^{\prime}, \rho^{\prime}\right)=\int_{0}^{1} d \lambda\langle U(\lambda)\rangle,
$$

where the average is taken over a MD simulation with the potential $U(\lambda)=\lambda U_{N N}-(\lambda-1) U_{r e f}$. The temperature and density were set to the experimental values at the melting point at normal conditions ${ }^{24}$. The reference system was chosen as an Einstein crystal for the solid and a Lennard-Jones fluid ${ }^{25}$ for the liquid. In the next step, the chemical potentials were evaluated by integrating the free energy as a function of density starting from $\rho^{\prime 26}$

$$
\begin{aligned}
\mu_{N N}\left(T^{\prime}, \rho\right) & =\frac{1}{N} F_{N N}\left(T^{\prime}, \rho^{\prime}\right)+b\left(T^{\prime}\right)\left(\ln \frac{\rho}{\rho^{\prime}}+1\right)+ \\
& +\frac{a\left(T^{\prime}\right)}{\rho^{\prime}}+c\left(T^{\prime}\right)\left(2 \rho-\rho^{\prime}\right)
\end{aligned}
$$

where $N$ is the number of particles in the simulation cell, and the parameters $a\left(T^{\prime}\right), b\left(T^{\prime}\right)$ and $c\left(T^{\prime}\right)$ were obtained by fitting the pressure dependence of the density using

$$
P\left(T^{\prime}, \rho\right)=a\left(T^{\prime}\right)+b\left(T^{\prime}\right) \rho+c\left(T^{\prime}\right) \rho^{2} .
$$

By equating the chemical potential of the two phases one obtains a transition pressure of -0.44 GPa at the chosen temperature $\mathrm{T}^{\prime}=998 \mathrm{~K}$. From the calculated ClausiusClapeyron equation $(d T / d P=6.85 \mathrm{~K} / \mathrm{GPa}$ from the calculated $\Delta S=\Delta E / T$ and $\Delta V$ on the theoretical melting 
line at $\mathrm{T}=998 \mathrm{~K}$ ) we then obtained the theoretical melting temperature at normal pressure which is $\mathrm{T}_{m}=1001$ $\mathrm{K}$.

We then analyzed the properties of the supercooled liquid below $\mathrm{T}_{m}$ by computing independently $\eta$ and $D$ in microcanonical MD simulations. The volume of the supercooled liquid was scaled with temperature according to the thermal expansion coefficient ( $\alpha$ is little dependent on temperature) given in Sec. II. We scaled the temperature from $1000 \mathrm{~K}$ to $500 \mathrm{~K}$ in eleven steps. At each step the system is equilibrated for $25 \mathrm{ps}$ with the thermostat. Overall the system is thus quenched from $1000 \mathrm{~K}$ to 500 $\mathrm{K}$ in $250 \mathrm{ps}$. At each temperature statistical averages are then collected on microcanonical simulations up to 2 ns long for the calculation of the viscosity as discussed below.

We first computed $D$ from the atomic mean square displacement on the time scale of $50 \mathrm{ps}$ on which the system does not crystallize at any temperature we considered. The values of $D$ as a function of temperature are reported in Fig. 11a. $D$ is still of the order of $10^{-6} \mathrm{~cm}^{2} / \mathrm{s}$ at the lowest temperature of $505 \mathrm{~K}$ considered here; it follows an Arrhenius behavior from $\mathrm{T}_{m}$ to $505 \mathrm{~K}$. The activation energy is $0.220 \pm 0.002 \mathrm{eV}$, a value much lower than the activation energy of $1.76 \mathrm{eV}$ obtained from the Arrhenius dependence of viscosity in GST measured in the temperature range $333-373 \mathrm{~K}$ (probably below $\mathrm{T}_{g}$ ) 27 . The ratio between the self-diffusion coefficient of $\mathrm{Ge}$ and Te $\left(D_{G e} / D_{T e}\right)$ increases by decreasing temperature as shown in Fig. 2, The values of $D$ obtained from the NN simulations were also validated by direct DFT molecular dynamics simulations at few selected temperatures with a small 216-atom cell (cf. Fig. 1 1 ). The DFT result at $1000 \mathrm{~K}$ is equal to that previously obtained in Ref $\underline{12}$ with the same cell and functional used here. We checked that the change of volume with temperature has a little effect on the diffusion coefficient as shown in Fig. Th which also reports the values of $D$ as a function of temperature once the density is fixed to the value at the melting point.

We then computed $\eta$ between $\mathrm{T}_{m}$ and a temperature $\mathrm{T}^{*}=700 \mathrm{~K}$ which turned out to be our crossover temperature by means of the Green-Kubo (GK) formula 23

$$
\eta=\frac{V}{k_{B} T} \int_{0}^{\infty}\left\langle\sigma_{x y}(t) \sigma_{x y}(0)\right\rangle d t,
$$

where $\sigma_{x y}$ is the off diagonal component of the stress tensor and $V$ the supercell volume. The integral in Eq. 4 is converged by restricting the integration time to $60 \mathrm{ps}$ due to the decay of the self-correlation function above $\mathrm{T}^{*}$. However, long simulation times up to 2 ns are needed to converge the average $(<.\rangle$.$) over different initial times$ $t=0$.

Above $\mathrm{T}^{*}$ the viscosity can be described by a simple Arrhenius (Fig. 1b) function with an activation energy of $0.17 \pm 0.035 \mathrm{eV}$, very close to the value of $0.2 \mathrm{eV}$ measured experimentally for the $\mathrm{Ge}_{0.15} \mathrm{Te}_{0.85}$ eutectic alloy above $\mathrm{T}_{m}{ }^{28}$. For the GeTe composition, experimental values of $\eta$ are available only at $1000 \mathrm{~K}$ yielding $\eta=2.59$ $\mathrm{mPa} \cdot \mathrm{s}$ which is twice as large as our result (cf. Fig. 1p). This discrepancy is not due to the NN potential but possibly to limitations of the underlying DFT framework. Previous works on $\mathrm{GeSe}_{2}$ have indeed shown that different choices of the exchange and correlation functional affect the dynamical properties of the liquid phase $\mathrm{e}^{29}$. The viscosity can be computed from the GK formula only above $\mathrm{T}^{*}$ since at lower temperatures the system crystallizes spontaneously on the time scale of few hundreds of ps which is not long enough to get the value of $\eta$ converged from Eq. 4. In the supercooled liquid, $\eta$ can not be defined on a time scale longer than the crystallization time which in GeTe is very short in the temperature range $500-700 \mathrm{~K}$.

We thus attempted to extrapolate $\eta$ below $\mathrm{T}^{*}$ by a VTF-like function with the constraint of matching the typical value of $10^{15} \mathrm{mPa} \cdot \mathrm{s}$ expected at $\mathrm{T}_{g}{ }^{7}$. Unfortunately, a reliable value of $\mathrm{T}_{g}$ for is not available from experiments because of the fast crystallization of $\mathrm{GeTe}$, and its theoretical estimate from simulations is difficult. Experimental data on $\mathrm{T}_{g}$ are available for the better glass formers $\mathrm{Ge}_{x} \mathrm{Te}_{(1-x)}$ alloys with $x=0.15-0.23 \underline{30}$. By a linear extrapolation with $\mathrm{x}$ of these latter data on $\mathrm{T}_{g}$ one obtains $\mathrm{T}_{g}=511 \mathrm{~K}$ for $x=0.5$ which is probably too high. On the other hand, $\mathrm{T}_{g}$ is customarily assumed to be slightly below the crystallization temperature, that is about 450 $\mathrm{K}$ in $\mathrm{GeTe}^{31}$. We then used the function proposed in ${ }^{32}$ that allows fitting $\eta$ over a wider range of temperatures

$$
\begin{gathered}
\log _{10} \eta(T)=\log _{10} \eta_{o}+\left(15-\log _{10} \eta_{o}\right) \\
\cdot \frac{T_{g}}{T} \exp \left[\left(\frac{m}{15-\log _{10} \eta_{o}}-1\right)\left(\frac{T_{g}}{T}-1\right)\right]
\end{gathered}
$$

where $m$ and $\eta_{o}$ are fitting parameters. In Eq. 5 $\eta=10^{15} \mathrm{mPa} \cdot \mathrm{s}$ at $\mathrm{T}_{g}$.

The parameter $m$ is is the fragility index of the supercooled liquid defined by the logarithmic derivative of $\eta$ at $\mathrm{T}_{g}, m=d\left(\log _{10} \eta\right) /\left.d\left(T_{g} / T\right)\right|_{T=T_{g}}$. We performed two fittings for two values of $\mathrm{T}_{g}$ as shown in Fig. 3. the first with $\mathrm{T}_{g}=450 \mathrm{~K}$ that yields $m=111\left(\log _{10} \eta_{o}=-0.18\right)$, and a second one with a somehow lower temperature $\mathrm{T}_{g}=400$ $\mathrm{K}$ which yields a very similar value of $m=104\left(\log _{10} \eta_{o}=-\right.$ 0.15). Similar results are obtained by using the modified VFT function proposed in Ref $\frac{33}{3}$. Our data on viscosity above $\mathrm{T}^{*}$ are thus consistent with a high fragility of the supercooled liquid. For sake of comparison we remark that $m=20$ in silica which is a typical strong liquid while $m=191$ in PVC which is a typical fragile liquid 34 . Unfortunately, due to the lack of data on $\eta$ below $\mathrm{T}^{*}$ and the uncertainties in the value of $\mathrm{T}_{g}$, we can not assign accurately the degree of fragility. Nevertheless, even for a value of $m$ as large as 100, the viscosity rises too steeply in the range 500-600 $\mathrm{K}$ to be consistent with the calculated values of $D$ and the application of SER. In fact, the SER actually breaks down at $\mathrm{T}<\mathrm{T}^{*}$ as discussed below.

In the hydrodynamic regime when the SER holds it is actually possible to estimate the viscosity on the shorter 

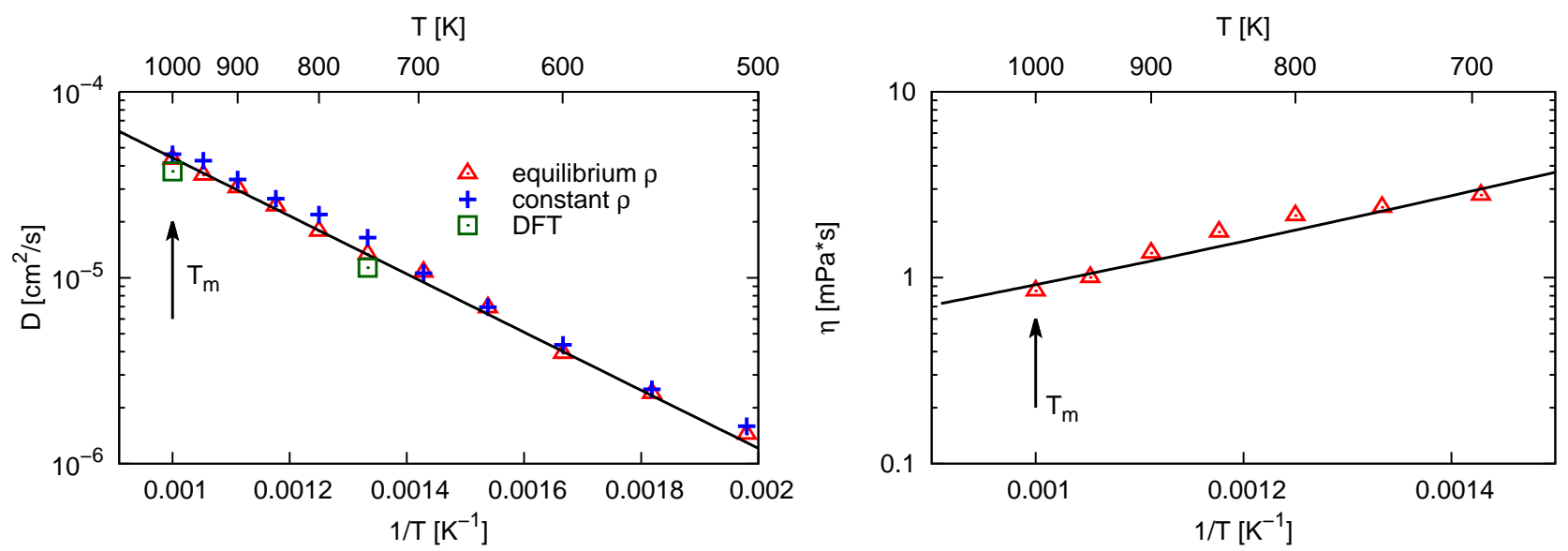

FIG. 1. a) Self-diffusion coefficient $D$ as a function of temperature in the supercooled liquid GeTe calculated from the mean square displacement (red triangles). The density is scaled with temperature according to the calculated thermal expansion coefficient. The open squares are the results of DFT simulations of a 216-atom cell. The crosses correspond to the values of $D$ computed by holding the density fixed to the value at the melting point. b) Calculated viscosity $\eta$ (Green-Kubo formula) as a function of temperature in the supercooled liquid. The density changes with temperature. The lines are Arrhenius fits of the data that give an activation energy of $0.220 \pm 0.002 \mathrm{eV}$ for $D$ and $0.17 \pm 0.035 \mathrm{eV}$ for $\eta$. T $\mathrm{T}_{m}$ is the theoretical melting temperature (see text).

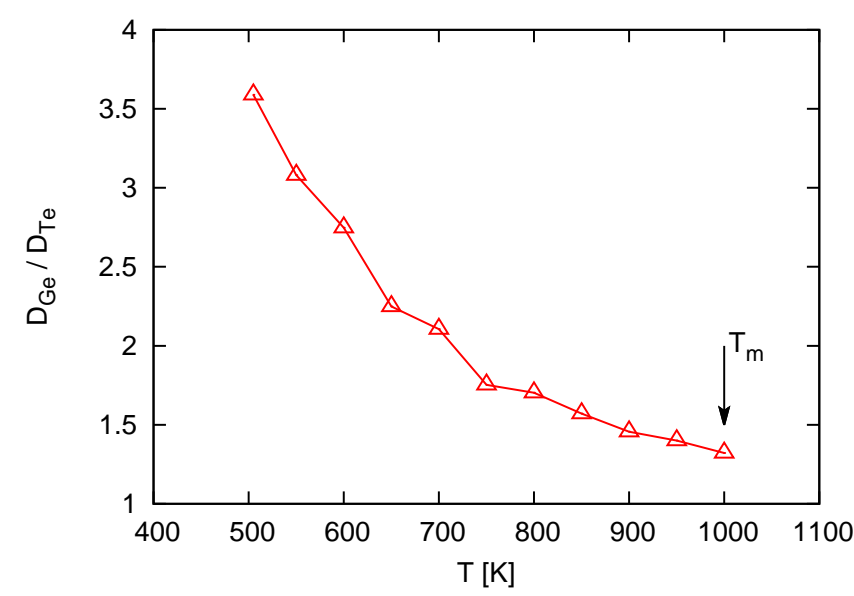

FIG. 2. Ratio of the self-diffusion coefficients of the two species $D_{G e} / D_{T e}$ as a function of temperature in supercooled liquid GeTe.

time scale of 50 ps by a finite size scaling analysis of the self-diffusion coefficient. The viscosity can be obtained from the scaling of $D$ with the edge $L$ of the cubic simulation cell as 35

$$
D(L)=D_{\infty}-\frac{2.387 k_{B} T}{6 \pi \eta L} .
$$

We considered three cubic models with 512, 1728 or 4096 atoms. By applying Eq. [6] above T*, we obtained values for $\eta$ very close to the GK data (Fig. 4) and consistent with the SER. However, when Eq. 6] is applied below $\mathrm{T}^{*}$, one obtains values of $\eta$ that are three orders of magnitude larger than those obtained from $D$ and the application of the SER $\left(\eta=\frac{k_{B} T}{6 \pi R D}\right.$, where $R$ is the average van der Waals radius of the two species) as shown in Fig. 4. This inconsistency demonstrates that the SER indeed breaks down. We remark that the numerical values of $\eta$ reported in Fig. 4 below $\mathrm{T}^{*}$ are not reliable since they are obtained from Eq. 6] which is not applicable when the SER breaks down.

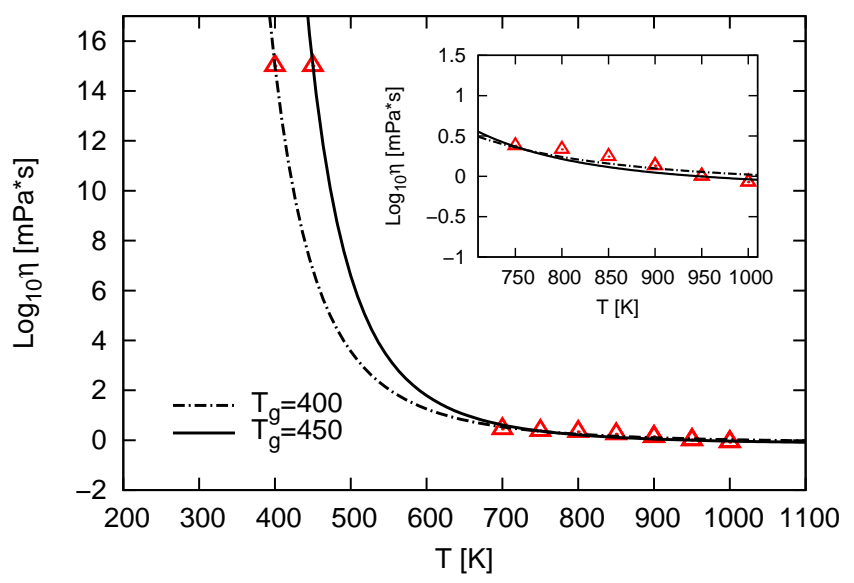

FIG. 3. Viscosity as a function of temperature in supercooled liquid GeTe. The lines are fittings with Eq. 5 of the GreenKubo data (triangles, cf. Fig. 10) above $\mathrm{T}^{*}$ under the constraint of reproducing the value of $\eta=10^{12} \mathrm{~Pa} \cdot \mathrm{s}$ at $\mathrm{T}_{g}$ with $\mathrm{T}_{g}=450 \mathrm{~K}$ (continuous line) or $\mathrm{T}_{g}=400 \mathrm{~K}$ (dashed line, see text). A magnification of the data for $\mathrm{T}>\mathrm{T}^{*}$ is shown in the inset. 


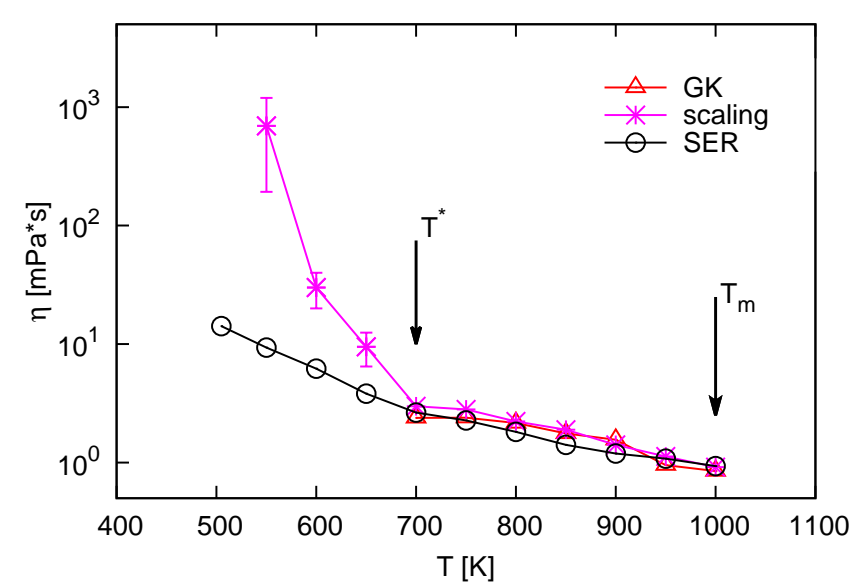

FIG. 4. Viscosity computed from the Green-Kubo (GK) formula (red triangles), from the scaling of the diffusion coefficient with the simulation cell size (stars, Eq. 6), and from the self diffusion coefficient of the 4096-atom cell and the application of the SER (open circles).

\section{CONCLUSIONS}

We have demonstrated by means of MD simulations that the supercooled liquid of the prototypical phase change compound GeTe shows a high atomic mobility ( $D$ $\sim 10^{-6} \mathrm{~cm}^{2} / \mathrm{s}$ ) down to temperatures very close to the glass transition temperature. Our calculated values of the viscosity as a function of temperature are consistent with a high fragility of the supercooled liquid. However, a compelling assessment of the degree of fragility would require a reliable estimate of $\mathrm{T}_{g}$ which is unfortunately unknown. The comparison between the calculated self diffusion coefficient and the viscosity demonstrates a breakdown of the SER below a crossover temperature of 700 K. These results support the experimental evidence of a breakdown of SER in the similar compound GST inferred by Orava et al. from ultrafast DSC measurements 9 . This feature is the key to understand the origin of the high crystallization rate in phase change memories. In the set process of PCM, the amorphous phase is heated above $\mathrm{T}_{g}$. Preliminary simulation results actually show that the glass transition experiences a hysteresis, the rapidly overheated amorphous phase differing from the supercooled liquid in a narrow range of temperature above $\mathrm{T}_{g}$. The overheated amorphous phase displays a breakdown of SER as well, although somehow less pronounced than in the supercooled liquid. This issue will be addressed in a future publication. However, in the set process of PCM, the temperature can rise significantly above $\mathrm{T}_{g}$ depending on the details of the programming pulses. Indeed optimized set pulses with peak intensities equal to the reset pulse (leading to the melting of the crystal) are implemented in current PCM devices ${ }^{36}$. Under these conditions the crystallization occurs from the supercooled liquid phase. In spite of a large viscosity, a high diffusivity is still possible in the supercooled liquid down to temperatures very close to $\mathrm{T}_{g}$ because of the breakdown of SER. This allows for the coexistence of a high diffusivity and a high driving force for crystallization that boost the crystallization speed at high supercooling. The crystallization of the supercooled liquid or of a highly mobile overheated amorphous phase might take place in a different manner with respect to the crystallization of the amorphous phase at temperatures below $\mathrm{T}_{g}$ which is of interest for data retention. The conclusion we can draw is that the self-diffusion coefficient in the supercooled liquid regime close to $\mathrm{T}_{g}$ can not be inferred from the viscosity and the application of the SER. Secondly, the measured Arrhenius behavior of the diffusivity and/or crystallization speed below $\mathrm{T}_{g}$ can not be extrapolated above $\mathrm{T}_{g}$ in the supercooled liquid. These results are of interest also for the refinement of the electrothermal modeling of PCM devices 37 .

\section{ACKNOWLEDGMENT}

This work has been partially supported by Italian MURST through the program Prin08, by the Cariplo Foundation through project MONADS and by Regione Lombardia and CILEA Consortium through a LISA Initiative (Laboratory for Interdisciplinary Advanced Simulation) 2011 grant [link: http://lisa.cilea.it]. We thankfully acknowledge the computational resources also by the ISCRA Initiative at Cineca (Casalecchio di Reno, Italy) and by CSCS (Manno, Switzerland).
* Corresponding author. Email address:

1 M. Wuttig and N. Yamada, Nat. Mater. 6, 824 (2007).

2 A. Pirovano, A. L. Lacaita, A. Benvenuti, F. Pellizzer, and R. Bez, IEEE Trans. Electron. Dev. 51, 452 (2004).

3 A.L. Lacaita and D.J. Wouters, Phys. Stat. Sol. A 205, 2281 (2008).

4 D. Lencer, M. Salinga, and M. Wuttig, Adv. Mat. 23, 2030 (2011).

5 S. Raoux, W. Welnic, and D. Ielmini, Chem. Rev. 110, 240 (2010).
6 S. R. Ovshinsky, Phys. Rev. Lett. 21, 1450 (1968).

7 C. A. Angell, Science 267, 1924 (1995); P. G. Debenedetti and F. H. Stillinger, Nature 410, 259 (2001).

8 D. Turnbull and J.J Fisher, J. Chem. Phys. 17, 71 (1949); D. Turnbull, J. Appl. Phys. 21, 1022 (1950).

9 J. Orava, A. L. Greer, B. Gholipour, D.W. Hewak and C. E. Smith, Nat. Mat. 11, 279 (2012).

10 G. Sosso, S. Caravati, J. Behler, and M. Bernasconi, Phys. Rev. B 85, 174103 (2012).

11 J. Behler, and M. Parrinello, Phys. Rev. Lett. 98, 146401 (2007). 
12 J. Akola, and R. O. Jones, Phys. Rev. B 76, 235201 (2007); Phys. Rev. Lett. 110, 205502 (2008).

13 R. Mazzarello, S. Caravati, S. Angioletti-Uberti, M. Bernasconi, and M. Parrinello, Phys. Rev. Lett. 104, 085503 (2010); erratum 107, 039902 (2011).

${ }^{14}$ P. Perdew, K. Burke, and M. Ernzerhof, Phys. Rev. Lett. 77, 3865 (1996).

15 S. Caravati, M. Bernasconi, T. D. Kühne, M. Krack, and M. Parrinello, Appl. Phys. Lett. 91, 171906 (2007).

16 RuNNer: A Neural Network Code for High-Dimensional Potential-Energy Surfaces, Jörg Behler, Lehrstuhl für Theoretische Chemie, Ruhr-Universität Bochum, Germany.

17 W. Smith and T. R. Forester, J. Mol. Graph. 14, 136 (1996).

18 G. Bussi, D. Donadio and M. Parrinello, J. Chem. Phys. 126, 014101 (2007)

19 S. Grimme, J. Comput. Chem. 27, 1787 (2006).

${ }^{20}$ V. M Glazov and O. D. Shelichov, Sov. Phys. Semicond. 18, 411 (1984).

21 J. VandeVondele, M. Krack, F. Mohamed, M. Parrinello, T. Chassaing, and J. Hutter, Comp. Phys. Comm. 167, 103 (2005); M. Krack, and M. Parrinello, High Performance Computing in Chemistry, edited by J. Grotendorst (NIC, Julich, 2004), Vol. 25, pp. 29-51; http://cp2k.berlios.de

22 S. Goedecker, M. Teter, and J. Hutter, Phys. Rev. B 54, 1703 (1996); M. Krack, Theor. Chem. Acc. 114, 145 (2005).

23 D. Frenkel and B. Smit, Understanding Molecular Simulation, Academic Press, San Diego, 1996.
24 W. Klemm, and G. Frischmuth, Z. anorg. Chem. 218, 249 (1934).

25 J. K. Johnson, J. A. Zollweg, and K. E. Gubbins, Mol. Phys. 78, 591 (1993).

26 L. M. Ghiringhelli, J. H. Los, E. J. Meijer, A. Fasolino, and D. Frenkel, Phys. Rev. Lett. 94, 145701 (2005).

27 J. A. Kalb, F. Spaepen, T.P. Leervad Pedersen, and M. Wuttig, J. App. Phys. 94, 4908 (2003).

28 F. Herwig and M. Wobst, Z. Metallkd. 82, 917 (1991).

29 M. Micolaut, R. Vuilleumier, and C. Massobrio, Phys, Rev. B 79, 214205 (2009).

30 D. Derewnicka, P. G. Zielinski, and H. A. davies, J. Mat. Sci. Lett. 1, 87 (1982).

31 S. Raoux, B. Munoz, H.-Y. Cheng, J. L. Jordan-Sweet, Appl. Phys. Lett. 95, 143118 (2009).

32 J. C. Mauroa, Y. Yueb, A. J. Ellisona, P. K. Guptac, and D. C. Allan, Proc. Natl. Acad. Sci. 106, 19780 (2009).

33 M. H. Cohen and G.S. Grest, Phys. Rev. B 20, 1077 (1979).

34 R. Böhmer, K. L. Ngai, C. A. Angell, and D. J. Plazek, J. Chem. Phys. 99, 4201 (1993).

35 B. Dünweg and K. Kremer, J. Chem. Phys. 99, 6983 (1993).

36 M. Borghi, P. Zuliani, E. Palumbo, G. Dalla Libera, M. Martinelli, and R. Annunziata, in Proceeding of the 12th European Phase Change and Ovonics Symposium (E\PCOS) 2012; available at www.epcos.org.

37 A. Redaelli, A. Pirovano, A. Benvenuti, and A. L. Lacaita, J. Appl. Phys. 103, 111101 (2008). 